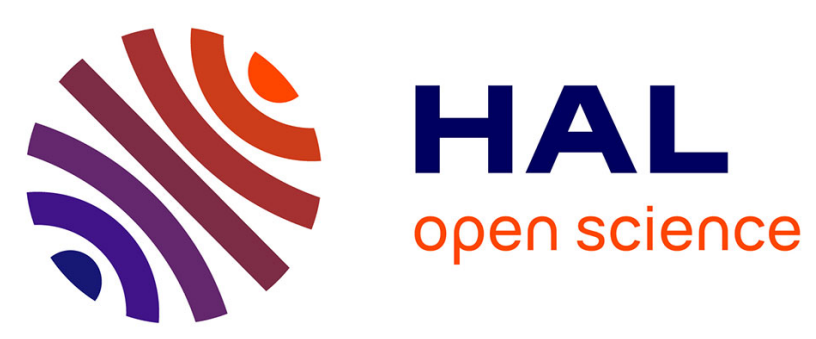

\title{
A feature extraction procedure based on trigonometric functions and cumulative descriptors to enhance prognostics modeling
}

Kamran Javed, Rafael Gouriveau, Noureddine Zerhouni, Patrick Nectoux

\section{- To cite this version:}

Kamran Javed, Rafael Gouriveau, Noureddine Zerhouni, Patrick Nectoux. A feature extraction procedure based on trigonometric functions and cumulative descriptors to enhance prognostics modeling. IEEE International Conference on Prognostics and Health Management, PHM'2013., Jan 2013, United States. pp.1-7. hal-00839903

\section{HAL Id: hal-00839903 https://hal.science/hal-00839903}

Submitted on 1 Jul 2013

HAL is a multi-disciplinary open access archive for the deposit and dissemination of scientific research documents, whether they are published or not. The documents may come from teaching and research institutions in France or abroad, or from public or private research centers.
L'archive ouverte pluridisciplinaire HAL, est destinée au dépôt et à la diffusion de documents scientifiques de niveau recherche, publiés ou non, émanant des établissements d'enseignement et de recherche français ou étrangers, des laboratoires publics ou privés. 


\title{
A feature extraction procedure based on trigonometric functions and cumulative descriptors to enhance prognostics modeling
}

\author{
Kamran Javed, Rafael Gouriveau, Noureddine Zerhouni, Patrick Nectoux \\ FEMTO - ST Institute, 24 rue Alain Savary, 25000 Besançon, France \\ Email: rafael.gouriveau@femto-st.fr
}

\begin{abstract}
Performances of data-driven approaches are closely related to the form and trend of extracted features (that can be seen as time series health indicators). 1) Even if much of datadriven approaches are suitable to catch non-linearity in signals, features with monotonic trends (which is not always the case!) are likely to lead to better estimates. 2) Also, some classical extracted features do not show variation until a few time before failure occurs, which prevents performing RUL predictions in a timely manner to plan maintenance task. The aim of this paper is to present a novel feature extraction procedure to face with these two problems. Two aspects are considered. Firstly, the paper focuses on feature extraction in a new manner by utilizing trigonometric functions to extract features (health indicators) rather than typical statistic measures like RMS, etc. The proposed approach is applied on time-frequency analysis with Discrete Wavelet Transform (DWT). Secondly, a simple way of building new features based on cumulative functions is also proposed in order to transform time series into descriptors that depict accumulated wear. This approach can be extended to other types of features. The main idea of both developments is to map raw data with monotonic features with early trends, i.e., with descriptors that can be easily predicted. This methodology can enhance prognostics modeling and RUL prediction. The whole proposition is illustrated and discussed thanks to tests performed on vibration datasets from PRONOSTIA, an experimental platform that enables accelerated degradation of bearings.
\end{abstract}

\section{INTRODUCTION}

Prognostics is the process of monitoring health of component or a system, and predicting the life time at which it is unable to perform the desired function. The predicted time indicates remaining useful life (RUL) of a degrading asset. According to ISO 13381-1, prognostics is defined as "the estimation of time to failure and risk for one or more existing and future failure modes" [1]. Mainly, prognostic forms part of prognostics and health management (PHM) discipline that links studies of failure mechanisms to system life cycle management. In context to health management, prognostics can be realized as a predictive maintenance strategy that avoids failure risks and inopportune spending to ensure availability, reliability and security of critical machinery [2]. As for examples, such critical machineries can be bearings, cutting tools, batteries or gears. The degradation of critical components can also affect other components of a system and cause major breakdowns or huge wastes.

According to literature, technical approaches for failure prognostics can be classified in three main groups: model-based, data-driven and hybrid approach [3]-[5]. Briefly, model-based approaches suppose that the degradation process can be formalized in mathematical and analytical form. Data-driven approaches for prognostics aim at transforming raw in situ data into appropriate information by performing non-linear modeling of real degrading systems for which it is very difficult to achieve analytical model. Data-driven approaches have an advantage of better applicability, when there is absence of prior knowledge or human experts. However, they can be less precise as compared to model-based approaches. Hybrid approaches for prognostics are combination of model-based and data-driven methods and benefit from both classes.

Although several approaches exist for prognostics, there is still a vacuum that is yet to be filled: nobody is able to a priori ensure that accurate prognostics model can be build (according to specific problem) [6]. Nevertheless, whatever the prognostic approach is, general steps have to be followed: (1) data acquisition, (2) feature extraction and selection, (3) prognostic modeling. Among these steps, feature extraction and selection is an essential step for prognostic modelling. Mainly, the degrading behavior of aging component or system is reflected by features that are derived from condition monitoring sensory data, for e.g., vibration, force and acoustic signals, temperature and voltage, etc. [7]. Irrespective to analysis domain (either time, frequency or both like wavelet transforms), the extracted features can be seen as time series health indicators that serve need of degradation modeling procedures.

Obviously, features that have monotonic trends that properly correlate to degradation phenomena may lead to simple and accurate prognostic models. Besides that, features that have non-monotonic trends and show ineffective failure progression phenomena can make prognostics more complicated and can lead to poor outcomes, therefore should be avoided. Recent works confirm that such essential characteristics as monotonicity and trendability of features are inevitable for prognostics modeling in order to accurately predict RUL of degrading asset [8]-[13]. This is the global aim of our work. Main contributions in this paper are as follows:

- Extract new features by applying trigonometric functions;

- Build new features based on cumulative function;

- Analyze fitness of features in terms of monotonicity and trendability. 
Paper is organized as follows. Section II highlights issues in existing feature extraction procedure that may lead to complex and inaccurate prognostics. Section III presents novel approach to extract features that have better fitness and can lead to enhanced prognostics. In section IV the entire proposition is illustrated by applying it to real vibration data of bearings and results are discussed. Finally, section V concludes this work.

\section{PROBlem FORMULATION}

Real industrial systems are intrinsically not "perfect" and usefulness of gathered data is highly dependent on variability phenomena, sensor non-linearity, etc. Transforming raw sensory data into features serve need of degradation modeling in prognostics. The extracted features can be seen as time series health indicators. However, the form of extracted degrading health indicators can be linear, non-linear, noisy or smooth, etc. Considering the fact that raw sensory data exhibit tremendous amount of hidden with noise, it can be quite challenging to obtain features that are monotonic and have trends that reflect failure progression in a meaningful way. Secondly, some classical extracted features like Root Mean Square (RMS) or Kurtosis, do not show any variation until a few time before failure occurs. Consequently, such situations prevent performing RUL prediction in a timely manner to plan maintenance task. According to this, some questions can be pointed out as meaningful for failure prognostics.

- How well the features reflect severity of failure progression?

- Do the feature have good trends that correlate to failure progression in meaningful way?

- How to judge suitability of features ?

Such questions can be easily understood by looking at a conceptual Fig. 1. The left part of this figure presents features that have attributes like high monotonicity, and have high trendability, as their trends properly correlate to failure progression in time $(\mathrm{t})$. In this figure, $t c$ indicates critical time to initiate prognostics.

As for example consider data-driven approaches: performances of such approaches are closely related to the form of the extracted features that are used to learn the behavior of degrading machinery. Features are extracted to make inference about the component or the system. Assuming that failure threshold is know a priori (this is not addressed in this paper), when models are learned and tested (i.e., from tc onwards) on data that consists of features that have "high" monotonicity
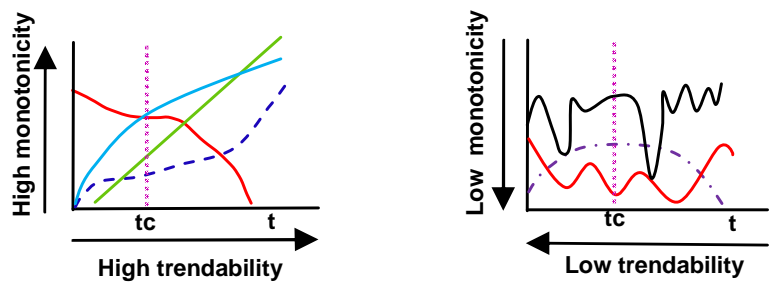

Fig. 1. Attributes of extracted features and trendability, prognostics modeling can be very simple and better RUL prediction can be achieved. But, if such models are exposed to features like those from the right part of Fig. 1, i.e. features that are non-linear trends showing "low" monotonicity and trendability attributes, the outcomes can be contrary. Also, even if much of data-driven approaches are suitable to catch non-linearity in signals, the accuracy of RUL prediction is still questionable. Therefore, it is strictly desired to extract features that can not only simplify prognostics modelling, but also lead to accurate RUL predictions. Next sections are dedicated to such issues.

\section{Cumulative FEATURES EXTRACTION PROCEDURE BASED ON TRIGONOMETRIC FUNCTIONS}

\section{A. Outline of the approach}

PHM approaches derive useful features directly from routinely monitored operational data. As with change of time wear in machinery increases, phenomena like excessive temperature, vibration or acoustic emission are observed [7], [14]. Prognostics approaches assume that the health indicators (features) are constant until a fault occurs in a component or system. However, real systems operate in dynamic environment, where rapid changes in machinery can take place. Although, feature extraction process transforms redundant health monitoring data into trends that reflect behavior of machinery. But obviously, one can not be sure that extracted trends properly reflect failure progression and serve need for prognostic modeling due to high variability phenomena.

As for example, consider case of bearings. They are the most common components in rotatory machinery and normally constitute a large percentage of failure cases in such machines. RUL prediction of such components is a challenging task for prognostic approaches that are based on vibration analysis [13]. As for such machinery vibration signal analysis is an important approach for condition monitoring, diagnostics or prognostics. But vibration signals are normally suppressed with noise and also subject to variations from a component or a system to another [13], [15]. In such situations, it is quite challenging for signal processing approaches like time domain, frequency domain or time-frequency (wavelet) analysis to furnish vibration based features that have monotonic trends. The main objective of proposed methodology is to extract features that reflect behavior of degrading asset in a meaningful way. In the first step, time-frequency analysis is performed on vibration data by applying discrete wavelet transform (DWT). Following that, features are extracted in a new manner by applying trigonometric functions and are smoothed further. A running total of each extracted feature is performed to build cumulative feature in order to have monotonic features with early trends. This approach can also be applied on classical features like: RMS, kurtosis, energy etc. Finally, multivariate data are analyzed for fitness to perform feature selection. The complete representation of this procedure is depicted in Fig. 2. 


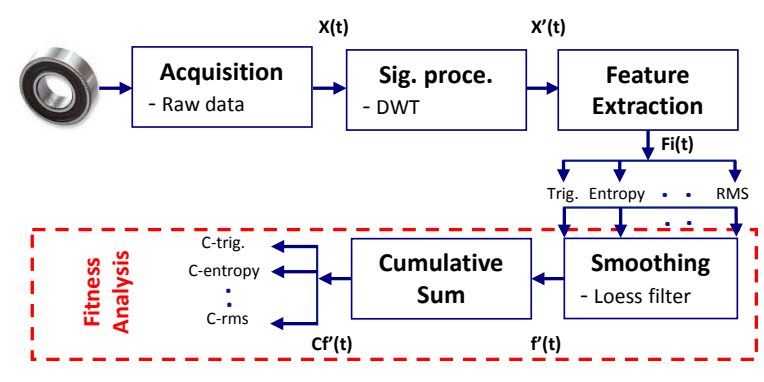

Fig. 2. Proposed Approach: from raw data to suitable features

\section{B. Feature extraction}

Vibration data acquired via sensors from rotating machinery (i.e., bearings) are redundant (too large) and highly nonlinear to be used directly. Therefore, it is desired to transform this data into relevant information known as features, that serve need of prognostics modeling. To achieve that, different signal processing approaches are hence applied to derive useful information about the machinery from such measurements. In simple words, extracted features are used to make inference about degrading machinery [16]. Mainly, signal processing methods like time domain, frequency domain or both (e.g., wavelet) are used for analysis of vibration data [17], [18].

Briefly, time domain analysis is directly performed on the time waveform. The time domain analysis is based on descriptive statistics like kurtosis, RMS, standard deviation, crest factor, etc. However, such time domain features are sensitive to oscillations in data. The frequency domain analysis transforms signal in frequency domain [19]. The most widely used approach in this category is Fast Fourier Transform (FFT). One of the main limitation of frequency domain approach is its inability to deal with non-stationary signals, which are the case in faulty machinery. To handle such requirements, time-frequency signal analysis has been developed. Several approaches of time-frequency domain are proposed in literature like Short Time Fourier Transform (STFT), Wigner-Ville Distribution (WVD), Wavelet Transform (WT) [3]. Within these approaches, WTs have gained great attention among researchers and industrials and are considered as an effective tool for non-stationary signals processing (like vibration), specially when it comes from rotating machinery like bearings [15], [18], [20], [21]. The next topic is dedicated to application of WT, that is discrete wavelet transform (DWT) in particular.

1) Discrete wavelet transform: In signal processing, WT is considered as an efficient approach to handle non-stationary vibration signals. It allows signal interpretation in both time and frequency domain. WT can be of two types: Continuous Wavelet Transform (CWT) and DWT. CWT has a drawback of impracticality with digital computers. Therefore, for practical reasons DWT is used. Mainly, DWT is achieved by discretization of CWT. Multi-resolution analyses is a useful implementation of DWT, where a discrete signal is passed through a low-pass filter that provides approximation coefficients and a high-pass filter that gives detail coefficients. In this manner, a time series signal is decomposed into two parts at first level of decomposition (i.e., approximation and detail). The time series signal can be further broken down into lower resolutions by successive decomposition of approximation part at current level [15], [20], [22]. As for an illustration, a 3-level wavelet decomposition is presented in Fig. 3.

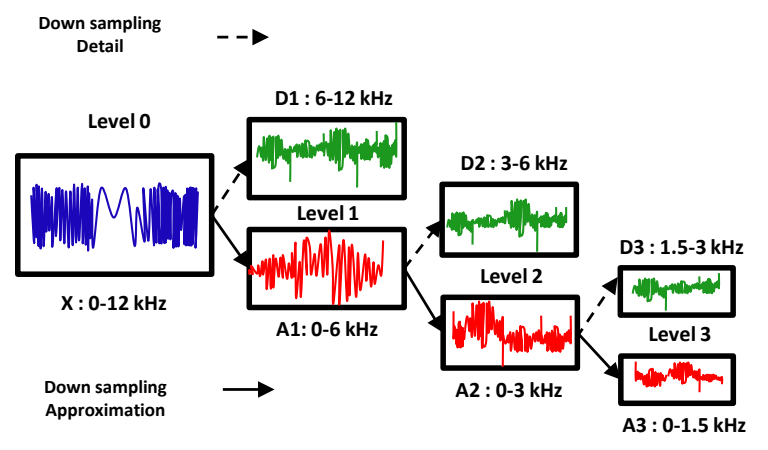

Fig. 3. DWT 3-level decomposition

According to above illustration, a useful property of DWT process is given in eq. 1 :

$$
X=A 3+D 1+D 2+D 3
$$

However, this task of multi-resolution analysis requires a priori information like type of mother wavelet, required level of decomposition and type of features to be extracted. It has been suggested in different works to use Daubichies wavelet of 4th order (D4) and to perform 3rd or 4th level of decomposition for bearing vibration signal [15], [21]. Hence, D4 and 4-level of decomposition was applied to acquired vibration data for bearing. Finally, choice of features is critical, typical features like RMS or kurtosis, can be highly sensitive to oscillations in data. Therefore, we propose a new way of extracting features by utilizing trigonometric functions that can have either monotonically increasing or decreasing forms, like the following: inverse hyperbolic cosine, inverse hyperbolic sine, inverse hyperbolic secant, etc. Such functions can produce features with low scale and have better trends. The approach can also be extended to other trigonometric functions as well. Different features extracted from 4th level approximation of vibration signal are summarizes in Table I.

TABLE I

FEATURES EXTRACTED FROM 4TH LEVEL APPROXIMATION

\begin{tabular}{l|l} 
Feature & Formula \\
\hline Std. of inverse hyperbolic cosine & $\sigma\left(\log \left[x_{i}+\left(x_{i}^{2}-1\right)^{1 / 2}\right]\right)$ \\
Std. of inverse hyperbolic sine & $\sigma\left(\log \left[x_{i}+\left(x_{i}^{2}+1\right)^{1 / 2}\right]\right)$ \\
Entropy (threshold) & $E(x)=\sum_{i} E\left(x_{i}\right)$ \\
Energy & $e=\sum_{i=0}^{n} x_{i}^{2}$ \\
Root mean square & $r m s=\sqrt{\frac{1}{n}\left(x_{1}^{2}+x_{2}^{2} \ldots+x_{n}^{2}\right)}$ \\
Skewness & $\frac{\sum_{i=1}^{n}\left(x_{i}-\bar{x}\right)^{3}}{(n-1) \sigma^{3}}$ \\
Kurtosis & $\frac{E(x-\mu)^{4}}{\mu^{4}}$ \\
Upper bound & $\max (x)+\frac{1}{2} \frac{\max (x)-\min (x)}{n-1}$ \\
\hline
\end{tabular}


2) Feature smoothing: Smoothing task is mainly performed to filter unwanted noisy part and to reduce the variability of extracted feature. Therefore each feature $F_{i}(t)$ from multivariate time series is smoothed to capture important trends in data by applying "LOESS" filter [23]. Mainly, "LOESS" is a popular smoothing method that is based on locally weighted regression function. Details can be found in [24].

\section{Cumulative Features}

The main idea in this step is to give condition indicators a form, that clearly reflects behavior of degrading asset. Generally the degradation of a machinery grows with time. Assuming that, components or systems do not undergo selfrecovery during operation eg. mechanical components like bearings, cutting tool, gears etc. Thereby, features that are extracted from such machinery should be monotonic and their trends should correlate with degradation. However, features with non-linear trends don't have clear indication about state of machinery under operation i.e., to distinguish among good, degrading or failure state. Besides that, such non-linearity can also represent self-healing. Therefore, features should have characteristics of monotonicity that reflect underlying positive or negative trends that correlate to failure progression. However, such assumption does not hold for batteries that may undergo self-repair during periods of non use [10]. Therefore, a simple strategy of building monotonic and trendable features with cumulative function is proposed which should be applied following classical procedure of feature extraction Fig. 2.

Mainly, it is a process of transforming a time series into feature that depict an accumulated form which can explicitly distinguish among states of machinery under observation. In simple words, a cumulative sum function furnishes a running total for a given time series (feature) (see eq. 2) as:

$$
C \grave{f_{n k}}=\frac{\sum_{i=1}^{n} \grave{f_{i k}}}{\sqrt{a b s\left(\sum_{i=1}^{n} \grave{f_{i k}}\right)}}, k=1,2 \ldots, N
$$

where, $C \grave{f}_{n k}$ represents running total of a "smoothed kth" feature $\grave{f}_{i k}$ up to "n" observations. It should be noted that, running or cumulative total of feature can be sensitive to noisy signal. So, it is better to perform feature smoothing a priori (see sec. III-B2). Finally, a cumulative feature can achieve better monotonicty and trendability that explicitly indicate failure progression (see Fig. 4).

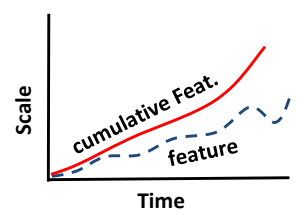

Fig. 4. Cumulative feature example

\section{Analyzing fitness of features}

This step aims at identifying subset of features that can have better fitness for further prognostics modeling. The central assumption is that, the features with attributes like monotonicity and trendability can lead to more accurate RUL predictions as compared to features that have contrary behavior. Therefore two simple measures are devised to assess suitability (or fitness) of a feature.

1) Monotonicity: Monotonicity characterizes underlying increasing or decreasing trend [10]:

$$
M=\left|\frac{n o . \text { of } d / d x>0}{n-1}-\frac{\text { no. of } d / d x<0}{n-1}\right|
$$

where " $n$ " is for number of observations in a particular feature. Monotonicity $M$ is measured by absolute difference of "positive" and "negative" derivatives for each feature. The value of $M$ can be from 0 to 1 : highly monotonic features will have $M=1$ and non-monotonic features $M=0$.

2) Trendability: Trendability can be related to functional form of an extracted feature and its correlation to time. That is, how the state of an asset varies with time. Surely, a constant function (of feature) will have zero correlation with time, where a linear function will have strong correlation. In similar way, correlation can vary with increase in nonlinearity (non-linear will result low correlation). To measure trendability a straight forward formula is given in eq. 4 .

$$
R=\frac{n\left(\sum x y\right)-\left(\sum x\right)\left(\sum y\right)}{\sqrt{\left[n \sum x^{2}-\left(\sum x\right)^{2}\right]\left[n \sum y^{2}-\left(\sum y\right)^{2}\right]}}
$$

$R$ called as correlation coefficient between two variables $\mathrm{x}$ and $y$ (i.e., feature and time index in our case). Correlation can be positive, negative, perfect or no correlation. Thus the value of $R$ can be from $-1<R<+1$.

\section{EXPERIMENTS AND RESULTS DISCUSSION}

In this section we illustrate the whole proposition on real data related to accelerated degradations of ball bearings from testbed PRONOSTIA. It should be noted that the data utilized in this paper were recently used for PHM challenge competition 2012 [25], for which participants could not provide satisfactory results mainly due to high noise and variability of data [26]. The detail about PRONOSTIA platform and experimental results are explained in following topics:

\section{A. PRONOSTIA testbed}

PRONOSTIA is an experimental platform which is dedicated to test and validate bearing fault detection, diagnosis and prognostics methods (Fig. 5). This testbed has been designed and realized at AS2M department of FEMTO-Institute. It enables accelerated degradations of bearings under constant and/or variable operating conditions, while gathering online health monitoring data (rotating speed, load force, temperature, vibration i.e., horizontal or vertical). 


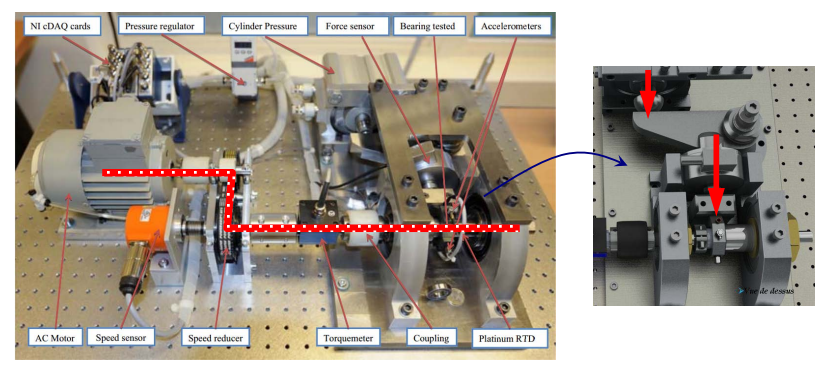

Fig. 5. PRONOSTIA bearing testbed

PRONOSTIA platform is composed of three main parts: a rotating part, a degradation generation part and measurement part. Therefore, main objective of this platform is to provide real data that characterize aging of ball bearings until total failure. Each degraded bearing contains almost all types of defects like balls, rings and cage. The data are recorded with specific sampling frequency that allows to capture all the frequency spectrum of bearing during degradation. The acceleration measures are sampled at $25.6 \mathrm{kHz}$ and the temperature measures are sampled at $0.1 \mathrm{~Hz}$. Bearing run-to failure experiments were stopped when amplitude of the vibration signal overpassed $20 \mathrm{~g}$. More details can be found in [26], [27].

Mainly, two different run-to failure data sets from different bearings (namely $B e r_{1-1}$ and $B e r_{1-6}$ ) under operating conditions $1800 \mathrm{rpm}$ and $4000 \mathrm{~N}$ are used to illustrate our proposition.

\section{B. Good case: Bearing 1-1}

The raw vibration data from $\mathrm{Ber}_{1-1}$ (Fig. 6) appears to be noisy. Secondly, it also has non-trendability behavior that can be easily observed from the envelop of vibration signal. Most of the portion of vibrations in an envelop show almost constant trends until 4th hour, but suddenly the vibrations grow rapidly from 4th hour onwards till the failure of bearing. Different features extracted from vibration signal are listed in Table I. When the quality of extracted features (indicated by solid lines in Fig. 6) is analyzed, one can notice that features like acosh, asinh and entropy show attributes of monotonicity and trendability which is not the case of all other features. Because, classical features like wave energy, Kurtosis are non-linear and do not explicitly reflect failure progression. Where, RMS and upper-bound show rapid increase in patterns when failure state occurs. Such features not only complicate prognostic modeling but can also affect RUL prediction accuracy.

To handle such issues, cumulative features are obtained following classical procedure of feature extraction (indicated by dotted lines in Fig. 6). Cumulative features are built in simple manner by performing running total on a particular feature (i.e, aggregating wear). Eventually, cumulative features obtained from all extracted features like asinh, RMS, entropy show high quality in terms of monotonicity and trendability even in case of non-linear features like entropy or kurtosis. Certainly such features (i.e. cumulative ones) can simplify prognostics modeling and possibly result accurate RUL predictions.

\section{Bad case: Bearing 1-6}

In case of $\operatorname{Ber}_{1-6}$ (Fig. 7) the vibration data envelop is highly noisy and also has high variability as compared to previous case (good case). Surely, from this kind of vibration data it is quite challenging to extract smooth and monotonic trends due to complicated nature of vibration signal. This problem can be observed in all types of extracted features (indicated by solid lines in Fig. 7). All the features show non-linear trends that do not reflect failure progression in a meaningful way. This can lead to poor prognostics outcomes, when such feature set are exposed to build RUL prediction models.

However, when running total of each feature time series is performed i.e., by aggregating wear values, all the cumulative features show good quality of trends in terms of monotonicity and trendability.

\section{Fitness analysis}

The, fitness analysis is performed on both dataset, i.e. good case and bad case, in order to highlight improvement made by our proposition of cumulative features in comparison to classical way of feature extraction. Therefore, monotonicity and trendability of features are measured . Obtained results are summarized in Table II, only in case of $B e r_{1-1}$, but a comparison of both cases is depicted in Fig. 8.

One can notice that, as compared to classical way of feature extraction, the results of cumulative features show high values of trendability $(R)$ and monotonicity $(M)$. That is, in both datasets (good case and bad case), cumulative features have higher fitness as compared to the classical ones. Surely, such findings benefit prognostics modeling.

TABLE II

FEATURES FITNESS ANALYSIS $\left(\operatorname{Ber}_{1-1}\right)$ :

\begin{tabular}{ccc|ccc} 
Feature $\left(\grave{f}_{i k}\right)$ & $R$ & $M$ & CumulativeFeat. $\left(C \grave{f}_{k}\right)$ & $R$ & $M$ \\
\hline acosh & 0,98 & 0,90 & C-acosh & 0,99 & 1 \\
asinh & 0,99 & 0,90 & C-asinh & 0,99 & 1 \\
entropy & 0,95 & 0,93 & C-entropy & 0,98 & 1 \\
energy & 0,81 & 0,43 & C-energy & 0,99 & 1 \\
rms & 0,91 & 0,90 & C-rms & 0,99 & 1 \\
skewness & 0,92 & 0,50 & C-skewness & 0,97 & 0,89 \\
kurtosis & 0,13 & 0,34 & C-kurtosis & 0,97 & 1 \\
upper bound & 0,85 & 0,79 & C-Up.bound & 0,99 & 1 \\
\hline
\end{tabular}

\section{CONCLUSION}

The main objective of prognostics modeling is accurate RUL prediction. Feature extraction is a critical step for all prognostic approaches. The application of most of the prognostics methods in literature is dependent on features that have attributes of monotonicity and trendability. This paper mainly, contributes to improve classical way of feature extraction. Firstly a new category of features is introduced in which 

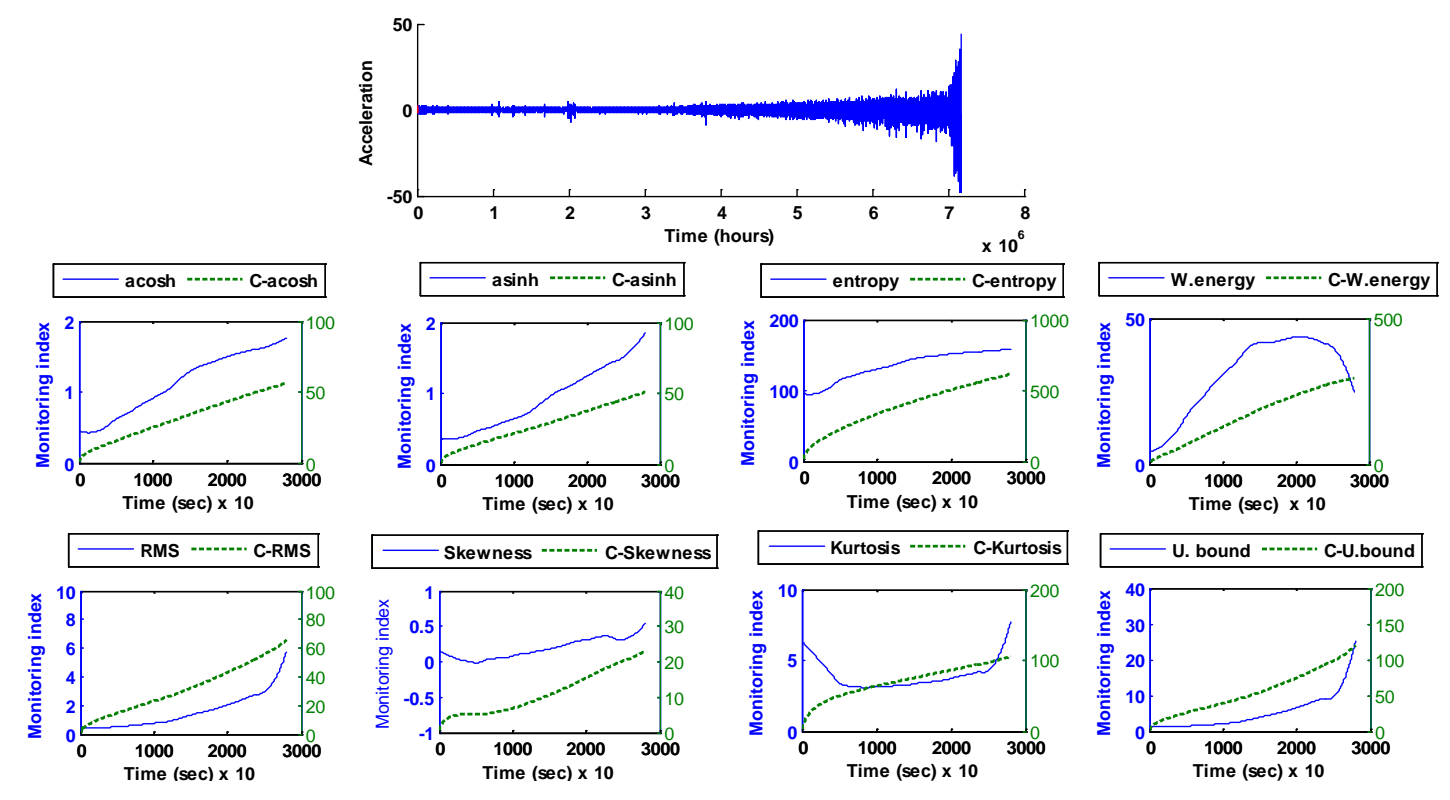

Fig. 6. Feature extraction (Good case)
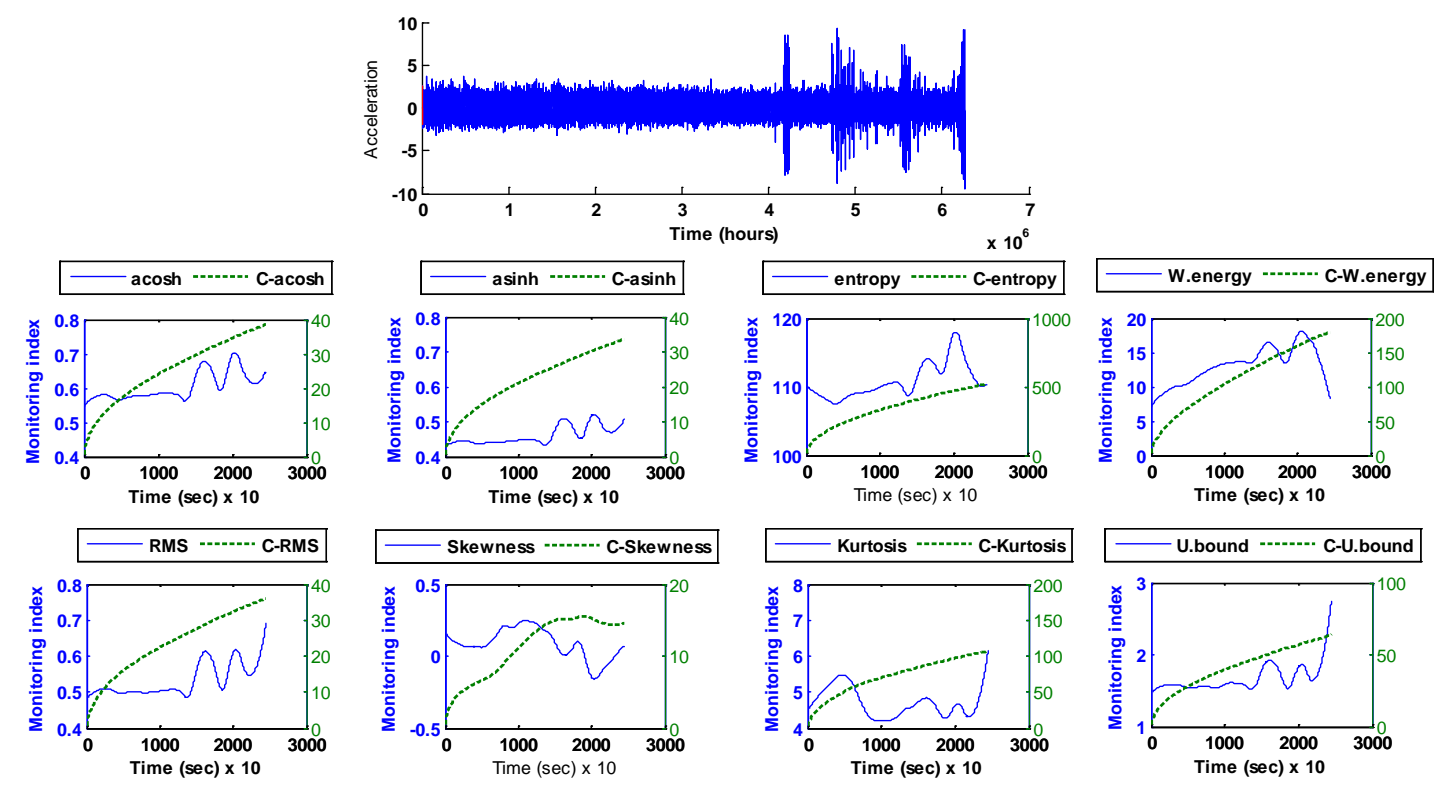

Fig. 7. Feature extraction (Bad case)

feature extraction is performed by utilizing trigonometric functions in a straight forward manner. Such features can be less sensitive to noise and variability of raw signal. However, this proposition needs to be further explored. But, as behavior of raw vibration signals vary from one case to another, one can not be sure that extracted features will have monotonic trends that reflect failure progression in a proper manner. To handle, this issue a simple solution is proposed. Mainly, this step aims at building cumulative features by performing running total on wear values of a particular feature. Proposed methodology is applied ro real data from PRONOSTIA platform (i.e., PHM challenge 2012). Obtained results show that cumulative feature have high monotonicity and trendability characteristics, that can surely enhance prognostics. Proposed approach can also be applied on classical features like RMS, kurtosis, skewness etc. The future perspective of this study will include datasets with different conditions and the final investigation on failure prognostics. 

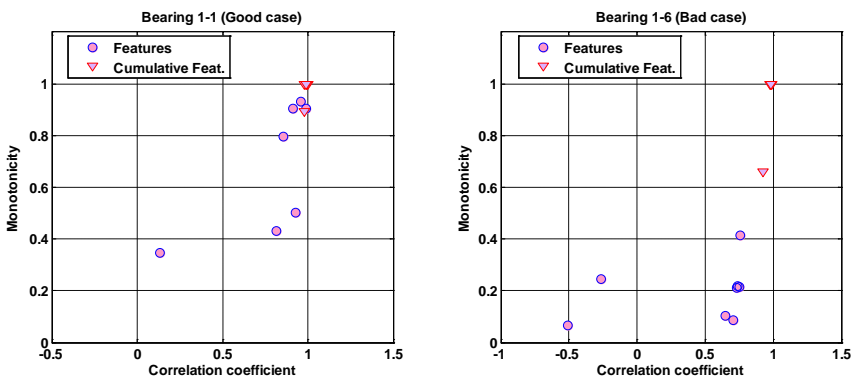

Fig. 8. Fitness Analysis

\section{REFERENCES}

[1] ISO13381-1, Condition monitoring and diagnostics of machines prognostics - Part1: General guidelines. Int. Standard, ISO, 2004.

[2] A.-S.-Y. Heng, "Intelligent prognostics of machinery health utilizing suspended condition monitoring data," Ph.D. dissertation, Queensland university,Australia, 2009.

[3] A. K. Jardine, D. Lin, and D. Banjevic, "A review on machinery diagnostics and prognostics implementing condition-based maintenance," Mechanical Systems \& Signal Processing, vol. 20, pp. 1483-1510, 2006.

[4] M. Lebold and M. Thurston, "Open standards for condition-based maintenance and prognostics systems," in 5th Annual Maint. and Reliability Conf., 2001.

[5] A. Heng and S. Zhang, "Rotating machinery prognostic: State of the art, challenges and opportunities," Mech. systems \& signal processing, vol. 23, pp. 724-739, 2009.

[6] K. Javed, R. Gouriveau, R. Zemouri, and N. Zerhouni, "Features selection procedure for prognostics: An approach based on predictability," in SAFEPROCESS'12, 8th IFAC Int. Symp. On Fault Dectection, Supervision and Safety for Tech. Proc., Mexico, 2012.

[7] S. Kumar and M. Pecht, "Modeling approaches for prognostics and health management of electronics," Int. Jour. of Performability Engr., vol. 6, no. 5, pp. 467-476, 2010.

[8] A. Mosallam, K. Medjaher, and N. Zerhouni, "Unsupervised trend extraction for prognostics and condition assessment," in A-MEST'12, 2nd IFAC workshop on Adv. Maint. Engr. Service and Technology, Spain, 2012.

[9] K. Medjaher, F. Camci, and N. Zerhouni, "Feature extraction and evaluation for health assessment and failure prognostics," in European Conference of the Prognostics and Health Management Society, Dresden, Germany, 2012.

[10] J. Coble and W. Hines, "Identifying optimal prognostic parameters from data:a genetic algorithms approach," in Annual Conference of the Prognostics and Health Management Society, USA, 2009.

[11] S. Porotsky and Z. Bluvband, "Remaining useful life estimation for systems with non-trendability behaviour," in Prognostics and Health Management (PHM), 2012 IEEE Conference on, USA, 20.

[12] E. Sutrisno, H. Oh, A. Vasan, and M. Pecht, "Estimation of remaining useful life of ball bearings using data driven methodologies," in Prognostics and Health Management (PHM), 2012 IEEE Conference on, USA, 2012.

[13] T. Wang, "Bearing life prediction based on vibration signals: A case study and lessons learned," in Prognostics and Health Management (PHM), 2012 IEEE Conference on, USA, 2012.

[14] T. Loutas and V. Kostopoulos, Utilising the Wavelet Transform in Condition-Based Maintenance: A Review with Applications, 2012. [Online]. Available: http://www.intechopen.com/books/

[15] S. B. Hocine Bendjama and and M. S. Boucherit, "Application of wavelet transform for fault diagnosis in rotating machinery," Int. Jour. of Machine Learning and Computing, vol. 2, no. 1, pp. 82-87, 2012.

[16] C. Nataraj and K. Kappanganthu, "Vibration-based diagnostics of rolling element bearings: state of the art and challenges," in 13th World Cong. In Mechanism and Machine Science, Mexico, 2012.

[17] W. Yan, H. Qiu, and N. Iyer, "Feature extraction for bearing prognostics and health management (phm)-a survey," in 62nd Meeting of the Society for Machinery, 2008.
[18] J. Zarei and J. Poshtan, "Bearing fault detection using wavelet packet transform of induction motor stator current," Tribology International, vol. 40, no. 5, pp. 763-769, 2007.

[19] J. Yu, "A hybrid feature selection scheme and self-organizing map model for machine health assessment," Applied Soft Computing, vol. 11, no. 5, pp. 4041-4054, 2011.

[20] J. Chebil, G. Noel, M. Mesbah, and M. Deriche, "Wavelet decomposition for the detection and diagnosis of faults in rolling element bearings," Jordan Journal of Mechanical and Industrial Engineering, vol. 3, no. 4, pp. 260-267, 2009.

[21] D. Tobon-Mejia, K. Medjaher, N. Zerhouni, and G. Tripot, "A datadriven failure prognostics method based on mixture of gaussians hidden markov models," Reliability, IEEE Transactions, vol. 61, no. 2, pp. 491503, 2012.

[22] S. G. MALLAT, "A theory for multiresolution signal decomposition:the wavelet representation," IEEE Transactions on pattern analysis and machine intellignece, vol. 11 , no. 7, pp. 674-693, 1989.

[23] Mathworks, "Curve fitting toolbox," 2010. [Online]. Available: http://mathworks.com/help/toolbox/curvefit/smooth.html

[24] J. Iwaniec, W. Lisowski, and T. Uhl, "Nonparametric approach to improvement of quality of modal parameters estimation," Jour. Of theoretical and applied mechanics, vol. 43, no. 2, pp. $327-344,2005$.

[25] Data-challenge, 2012. [Online]. Available: http://www.femto-st.fr/ ieee-PHM2012-data-challenge

[26] P. Nectoux, R. Gouriveau, K. Medjaher, E. Ramasso, B. Morello, N. Zerhouni, and C. Varnier, "Pronostia: An experimental platform for bearings accelerated life test," in IEEE International Conference on Prognostics and Health Management, USA, 2012.

[27] "Ieee phm 2012 prognostic challenge." [Online]. Available: http: //www.femto-st.fr/f/d/IEEEPHM2012-Challenge-Details.pdf 